\title{
VALIDACIÓN DE LA ESCALA DE MORISKY DE 8 ÍTEMS EN PACIENTES CON ENFERMEDAD RENAL CRÓNICA
}

\author{
NINFA MARLEN CHAVES TORRES ${ }^{1}$, JORGE ENRIQUE ECHEVERRI SARMIENTO ${ }^{2}$, \\ DAVID ANDRÉS BALLESTEROS ${ }^{3}$, JECXY QUIJANO RODRIGUEZ ${ }^{4}$, DANIELA CAMACHO ${ }^{4}$ \\ ${ }^{1}$ Md, MSc. Farmacología, docente facultad de medicina UMNG. \\ ${ }^{2} \mathrm{Md}$, Internista, Nefrólogo, Unidad Renal Hospital Militar Central \\ ${ }^{3} \mathrm{Md}$, Internista, Fellow de Nefrología UMNG \\ ${ }^{4} \mathrm{Md}$. Cirujano \\ Correspondencia: Ninfa Marlen Chaves Torres \\ E-mail: marlenchaveztdoc@yahoo.es \\ Dirección: Dirección: Transv. 3 No. 49-00. Piso 6 \\ Teléfono: 6500000,6343200 Ext 2160/2048 \\ Bogotá DC - Colombia
}

Recibido: 5 Febrero 2016 Aceptado: 6 Julio 2016

\section{RESUMEN}

Introducción: Es importante proveer un adecuado y oportuno manejo a largo plazo de la ERC, por medio de estrategias farmacológicas y no farmacológicas, sin embargo, la baja adherencia de los pacientes al tratamiento farmacológico pone en riesgo la efectividad de la intervención.

Objetivo: Traducir al español, validar y adaptar la escala de adherencia al tratamiento de Morisky de 8 items a pacientes con ERC.

Materiales y Métodos: La traducción del MMAS-8 se realizó de acuerdo con las directrices para la traducción y la adaptación de instrumentos de medida para ser utilizados en investigación médica, se validó mediante juicio de expertos siguiendo el método Delphi. Se realizó un estudio piloto corte transversal, donde se encuesto a 30 pacientes que acudieron a consulta de predialisis de la unidad renal del hospital militar central en Bogotá Colombia.

Resultados: La escala MMAS-8 traducida al español fue sometida a los jueces para su validación obteniéndose un porcentaje de acuerdo igual o mayor al $80 \%$ para todas las preguntas, se calculó el coeficiente de concordancia $\mathrm{W}$ de Kendall que en este caso fue 0,8 con un p valor de 0,0001 y se interpretó como un alto grado de concordancia entre las valoraciones hechas por los jueces a los diferentes ítems del cuestionario. Se aplicó el MMAS-8 a 30 pacientes con una media de edad de 75,9 años (+/-15), el nivel de adherencia al tratamiento se analizó según el puntaje de la escala de Morisky de 8 items, clasificándose como baja adherencia al tratamiento en el $62,5 \%$ de los casos con puntaje menor de 6 , mediana adherencia $28,1 \%$ de los pacientes puntaje entre 6 y 7 y alta adherencia solo el $9,4 \%$ de los pacientes con puntaje mayor de 8.

Conclusiones: La escala de medición de adherencia al tratamiento de Morisky de 8 items es válida para medir la adherencia al tratamiento farmacológico de pacientes con enfermedad renal crónica según juicio de expertos.

Palabras claves: Cumplimiento de la Medicación, Insuficiencia renal crónica, Validación 


\title{
VALIDATION OF THE MORISKY 8 ITEMS SCALE IN PATIENTS WITH CHRONIC KIDNEY DISEASE
}

\begin{abstract}
Introduction: It is important to provide long-term, adequate and timely treatment of the Chronic kidney disease, by pharmacological and non-pharmacological strategies, however, low adherence of patients to drug treatment puts at risk the effectiveness of the intervention.

Objective: Translate to Spanish, validate and adapt the scale of adherence Morisky 8 items patients with chronic kidney disease.

Materials and methods: Translation from MMAS- 8 was performed according to the guidelines for the translation and adaptation of measuring instruments for use in medical research. It was validated by expert judgment following the Delphi method. A pilot study was conducted cross-sectional, where 30 patients who consulted predialysis renal unit of the central military hospital in Bogota Colombia was surveyed.

Results: MMAS-8 Scale translated into Spanish was submitted to the judges for validation, obtaining a percentage of agreement equal to or greater than $80 \%$ for all questions, the coefficient of Kendall $\mathrm{W}$ in this case was 0.8 with a p value of 0.0001 was calculated and it is interpreted as a high degree of consistency between the assessments made by the judges to the different items of the questionnaire. MMAS- 8 is applied to 30 patients with a mean age 75.9 years $(+/-15)$, the level of adherence to treatment is performed according to the scale score Morisky 8 items, classified as low adherence to treatment in $62.5 \%$ of cases with lower score of 6 , adhesion median $28.1 \%$ of patients score between 6 and 7, and high adhesion only $9.4 \%$ of patients with major score of 8 .
\end{abstract}

Conclusions: The scale measuring adherence Morisky 8 items It is valid to measure adherence to drug treatment of patients with chronic kidney disease according to expert judgment.

Keywords: Medication Adherence, Chronic renal insufficiency, Validation. 


\section{VALIDAÇÃO DA ESCALA DE MORISKY 8 EM PACIENTES COM DOENÇA DO RENAL CRÔNICA}

\section{RESUMO}

Introdução: É importante fornecer um tratamento a longo prazo, adequado e oportuno da doença renal crónica, através de estratégias farmacológicas e não farmacológicas, no entanto, a baixa adesão dos doentes ao tratamento medicamentoso põe em risco a eficácia da intervenção.

Objetivo: Traduzir para o espanhol, validar e adaptar a escala de adesão Morisky 8 itens pacientes com doença renal crônica.

Materiais e métodos: A tradução do MMAS-8 foi realizada de acordo com as diretrizes para a tradução e adaptação de instrumentos de medição para uso em uma investigação médica. Foi validado por um juízo especialista seguindo o método Delphi. Um estudo piloto foi realizado em corte transversal, onde foram entrevistados 30 pacientes que consultaram a unidade pré-diálise renal do hospital militar central em Bogotá Colômbia.

Resultados: A escala de MMAS-8 traduzida para o espanhol foi submetida aos juízes para validação, obtendo-se uma percentagem de concordância igual ou superior a $80 \%$ para todas as questões, o coeficiente de Kendall W neste caso 0,8 com um valor de $\mathrm{p}$ de 0,0001 foi calculado $e$ interpretado como um alto grau de consistência entre as avaliações feitas pelos juízes aos diferentes itens do questionário. O MMAS- 8 é aplicado a 30 pacientes com idade média de 75,9 anos (+/- 15), o nivel de aderência ao tratamento é realizado de acordo com o escore Morisky 8 itens, classificados como baixa adesão ao tratamento em $62,5 \%$ dos casos com Menor pontuação de 6 , adesão mediana $28,1 \%$ dos pacientes pontuação entre 6 e 7, e alta adesão apenas 9,4\% dos pacientes com pontuação maior de 8.

Conclusões: A adesão escala de medição para tratamento Morisky 8 itens é válido para medir a adesão ao tratamento medicamentoso de pacientes com doença renal crônica, de acordo com o julgamento cientistas de investigação.

Palavras-chave: Adesão à medicação Insuficiência renal crônica, validação. 


\section{Introducción}

La enfermedad renal crónica (ERC), se define como anormalidades estructurales o funcionales del riñón por más de 3 meses, con implicaciones para la salud y se clasifica basada en la causa, tasa de filtración glomerular y niveles de albuminuria (1-2). En Colombia según el Instituto Nacional de Salud, la ERC tuvo una incidencia estimada de 13 por 100.000 en el 2011 , con una prevalencia del $0.43 \%$ y una tasa de mortalidad 4.68 por 100.000 habitantes, constituyendo la octava causa de enfermedad no transmisible en Colombia. Por lo tanto, hoy en día es considerada un problema de salud pública (3).

Numerosos estudios han sugerido la asociación entre ERC y riesgo cardiovascular entre ellos, el de Hypertension Detection and Follow-up Program y el de Hypertension Optimal Treatment (HOT), mostraron que el aumento de la creatinina sérica es un importante predictor de riesgo de muerte por evento cardiovascular en estos pacientes. El estudio Prevention of Renal and Vascular End-Stage Disease (PREVEND) encontró asociación entre la microalbuminuria y la mortalidad tanto global como cardiovascular en la población general, con estos hallazgos se evidencian la importancia de proveer un adecuado y oportuno manejo a largo plazo de la ERC, buscando mejorar el pronóstico cardiovascular en estos pacientes $(4,5)$. Es así como la terapia farmacológica está orientada a controlar las patologías asociadas y otros posibles desencadenantes buscando prevenirla (6).

Mientras que en los pacientes con diagnóstico de ERC el objetivo del tratamiento es mejorar los síntomas, disminuir la progresión y las complicaciones de la enfermedad, buscando una mejor calidad de vida para estos pacientes, que se lleva a cabo por medio de estrategias farmacológicas y no farmacológicas, sin embargo, la baja adherencia de los pacientes al tratamiento farmacológico pone en riesgo la efectividad de la intervención.

La Organización Mundial de la Salud (OMS) basada en las definiciones de Haynes y Rand define la adherencia al tratamiento prolongado como el grado en que el comportamiento de una persona en este caso: tomar el medicamento, seguir un régimen alimentario y ejecutar cambios en el modo de vida, se corresponde con las recomendaciones acordadas con el prestador de asistencia sanitaria (1).
Es así entonces, como los pacientes deben ser "socios activos" de los médicos en su propia atención, donde la comunicación es un requisito esencial para la buena práctica clínica. Existen múltiples factores, más de 250 según Haynes et al, que intervienen en el incumplimiento del manejo farmacológico por parte del paciente, entre ellos, se encuentran la dependientes de la estructura sanitaria o empresa prestadora de servicios, la enfermedad y el tratamiento establecido (7). Así pues, la detección de los pacientes no adherentes y de las posibles causas de incumplimiento permite identificar, prevenir y resolver los problemas relacionados a dosis, pautas y duración del tratamiento farmacológico, y de esta manera llegar a evitar parte de los problemas relacionados con medicamentos (PRM) (8).

Los instrumentos de medición de la adherencia terapéutica se han divido en dos categorías: Los métodos directos de la toma de la medicación tales como los registros de farmacia, conteo de píldoras, la vigilancia electrónica y los niveles en plasma sanguíneo; y los métodos indirectos del uso de fármacos en el paciente a través de informes, entrevistas o cuestionarios (7). Aún no se ha podido establecer qué tipo de herramienta es más efectiva para medir la adherencia y no ha sido posible demostrar que hay una herramienta, aislada o en conjunto, que sea totalmente eficaz en todos los pacientes, enfermedades o entornos (9). Sin embargo, The 8-item Morisky Medication Adherence Scale (MMAS-8), mediante una entrevista personalizada sencilla permite hacer un estudio cualitativo y cuantitativo de la adherencia al tratamiento; y ubica a los pacientes en una escala de alta, media o baja adherencia (10).

El cuestionario para medir la adherencia de Morisky de 8 items fue validado por Morisky en el 2008 en paciente con hipertensión arterial, en el 2012 fue aplicado en Francia en pacientes con hipertensión, en el 2013 en China en pacientes con Diabetes mellitus en el año 2014 en Brasil a pacientes con hipertensión y una validación en versión China para paciente con epilepsia (11-14). Lo cual evidencia propiedades psicométricas adecuadas para evaluación de la falta de adherencia al tratamiento en pacientes con diferentes patologías crónicas. El propósito de este trabajo es traducir al español, validar y adaptar la escala de adherencia al tratamiento de Morisky de 8 items a pacientes con ERC. 


\section{Metodología}

\section{Traducción del instrumento}

El MMAS-8 fue traducido al español bajo la vigilancia del autor del MMAS-8 de acuerdo con las directrices para la traducción y la adaptación de instrumentos de medida para ser utilizados en investigación médica. Se seleccionaron, 3 personas expertas en traducción de documentos médicos. La primera tomó el cuestionario original en inglés (v1) y lo tradujo al español (v2). La segunda tomó esta traducción al español (v2) y la tradujo de nuevo al inglés (v3). La tercera persona compara esta versión en inglés (v3) con la versión original y determinó, enunciado por enunciado, que estos eran equivalentes en significado, a su vez dos de los investigadores sugirieron pequeñas modificaciones en algunos enunciados para hacerlo más comprensible para el paciente colombiano con ERC, se discutieron estos ajustes con los traductores quienes estuvieron de acuerdo en que no alteraban la semántica del cuestionario, generando así la versión final del cuestionario en español adaptada para pacientes con enfermedad renal crónica (15).

\section{Validación del instrumento}

Para asegurar que el MMAS-8 traducido al español y adaptado para medir adherencia en pacientes colombianos con enfermedad renal crónica, conserva las mismas propiedades métricas que la versión en inglés, se sometió a juicio de expertos siguiendo el método Delphi, se seleccionaron expertos que actuarían como jueces, la selección se realizó teniendo en cuenta la formación académica y experiencia relacionada con procesos de seguimiento farmacoterapéutico y manejo de pacientes con enfermedad renal crónica. Con los siguientes perfiles:

I. Químico farmacéutico, magister en farmacia hospitalaria, con amplia experiencia en farmacia hospitalaria, seguimiento farmacoterapéutico, farmacovigilancia, docencia universitaria e investigación, con publicaciones nacionales e internacionales en área de farmacia hospitalaria, seguimiento farmacoterapéutico y farmacovigilancia.

II. Médico, especialista en epidemiologia, magister en ciencias farmacología, con experiencia en farmacología clínica, farmacovigilancia, farmacoeconomía. Publicaciones en estas áreas de experticia.
III. Enfermera, especialista en cuidados intensivos, docencia universitaria, magister en ciencias farmacología, experiencia en cuidos intensivos, seguimiento a pacientes con patologías crónicas, seguimiento farmacoterapéutico, publicaciones en estas áreas del conocimiento.

IV. Médico, magister en ciencias farmacología, con experiencia en atención primaria, farmacovigilancia, coordinación del grupo de apoyo a las salas especializada de medicamentos del INVIMA. Publicaciones en farmacoepidemiología y farmacovigilancia.

V. Médico, especialista en medicina interna - nefrología, con experiencia en la atención de pacientes con enfermedad renal crónica. Con publicaciones nacionales $e$ internacionales en el área de la Nefrología clínica.

Se realizó una reunión investigadora principal y jueces, donde se explicó el objetivo y alcances del proyecto, objetivo del juicio de expertos, y lo que se pretende medir con cada uno de los ítems de la encuesta. Se proporcionó información suficiente sobre el uso que se pretende dar a la encuesta de medición de adherencia en pacientes con enfermedad renal crónica. Se envió vía correo electrónico una plantilla previamente diseñada que incluye los ítems a evaluar, los rangos de valoración y los objetivos de la investigación, a los jueces se les pedio que evalúen la equivalencia entre el cuestionario de Morisky de 8 items en inglés y el mismo cuestionario traducido al español y adaptado a pacientes con enfermedad renal crónica, calificando cada ítem por separado, como muy adecuado, bastante adecuado, adecuado, poco adecuado e inadecuado. Se digitaron estos datos en una base de datos de Excel se calculó el porcentaje de acuerdo de los expertos para cada items y si este porcentaje es menor al $80 \%$ se enviaran las respuestas obtenidas nuevamente a todos los expertos y se les preguntara en una segunda ronda si desean mantener su calificación inicial o prefieren cambiar de opinión al conocer las respuestas de los demás jueces. Los datos obtenidos de los expertos se procesaron en el paquete estadístico SPSS en el que se realizó un análisis estadístico de concordancia.

Se realizó un estudio piloto corte transversal, donde se encuesto a 30 pacientes que acudieron a consulta de predialisis de la unidad renal del hospital militar central en Bogotá Colombia, que estuvieran recibiendo tratamiento farmacológico, entre enero y junio de 2015. Los participantes fueron reclutados al azar durante el horario regular de la consulta con el médico tratante. Todos los pacientes fueron informados del objetivo del estudio $y$ 
manifestaron su consentimiento por escrito antes de su inclusión en el estudio, que fue aprobado por el comité de ética del hospital militar central.

\section{Recolección de datos}

Los datos fueron recolectados a través de entrevistas durante la consulta de control en el programa de predialisis, realizada por el médico tratante previamente informado sobre el objetivo del estudio, la forma adecuada de aplicación de la encuesta MMAS-8 y la recolección de los datos. Se incluyeron datos sociodemográficos y algunos parámetros paraclínicos que constituyen metas terapéuticas, la adherencia al tratamiento se catalogó según el puntaje obtenido al aplicar la escala MMAS-8 como alta adherencia cuando obtu- vo 8 puntos, media si obtuvo de 6 ó 7 puntos y baja si obtuvo 5 puntos o menos.

\section{Análisis estadístico}

Se construyó una base de datos en Excel ${ }^{\circledR}$ para importarla al paquete estadístico SPSS y se realizó el análisis basado en distribuciones de frecuencia, medidas de tendencia central y de dispersión según la naturaleza cualitativa o cuantitativa de las variables.

\section{Resultados}

Versión en español de Colombia, adaptada para pacientes renales de la escala de adherencia al tratamiento de Morisky de 8 items (MMAS-8) (tabla 1)

Tabla 1. Escala de adherencia al tratamiento de Morisky de 8 items, traducida al español y adaptada para pacientes con enfermedad renal crónica.

\begin{tabular}{c|l|l}
\hline No item & \multicolumn{1}{|c}{ Ítem ingles } & \multicolumn{1}{c}{ Ítem español } \\
\hline 1 & Do you sometimes forget to take your medicine? & ¿A veces se olvida de tomar su medicamento? \\
\hline 2 & $\begin{array}{l}\text { People sometimes miss taking their medicines for } \\
\text { reasons other than forgetting. Thinking over the } \\
\text { past } 2 \text { weeks, were there any days when you did } \\
\text { not take your medicine? }\end{array}$ & $\begin{array}{l}\text { La gente a veces omite tomar sus medicamentos por } \\
\text { razones diferentes al olvido. Pensando en las últimas } \\
2 \text { semanas, chubo algún día que no toma su medica- } \\
\text { mento? }\end{array}$ \\
\hline 3 & $\begin{array}{l}\text { Have you ever cut back or stopped taking your } \\
\text { medicine without telling your doctor because you } \\
\text { felt worse when you took it? }\end{array}$ & $\begin{array}{l}\text { ¿Alguna vez ha recortado o dejado de tomar el } \\
\text { medicamento sin consultar antes con su médico, ya } \\
\text { que se sintió peor cuando lo tomaba? }\end{array}$ \\
\hline 5 & $\begin{array}{l}\text { When you travel or leave home, do you some- } \\
\text { times forget to bring along your medicine? }\end{array}$ & $\begin{array}{l}\text { Cuando viaja o sale de casa, ¿a veces se olvida de } \\
\text { llevar su medicina? }\end{array}$ \\
\hline 6 & $\begin{array}{l}\text { Did you take all your medicines yesterday? } \\
\text { When you feel like your symptoms are under con- } \\
\text { trol, do you sometimes stop taking your medicine? }\end{array}$ & $\begin{array}{l}\text { Cuando siente que sus sintomas están bajo control, } \\
\text { ¿a veces deja de tomar su medicamento? }\end{array}$ \\
\hline 7 & $\begin{array}{l}\text { Taking medicine every day is a real inconvenience } \\
\text { for some people. Do you ever feel hassled about } \\
\text { sticking to your treatment plan? }\end{array}$ & $\begin{array}{l}\text { Tomar medicamentos todos los días es un verdadero } \\
\text { inconveniente para algunas personas. ¿Alguna vez } \\
\text { se siente presionado a apegarse a su plan de trata- } \\
\text { miento? }\end{array}$ \\
\hline 8 & $\begin{array}{l}\text { How often do you have difficulty remembering to } \\
\text { take all your medicine? } \\
\text { A. Never/rarely } \\
\text { B. Once in a while } \\
\text { C. Sometimes } \\
\text { D. Usually } \\
\text { E. All the time }\end{array}$ & $\begin{array}{l}\text { ¿Con qué frecuencia tiene dificultad para acordarse } \\
\text { de tomar todos sus medicamentos? } \\
\text { Respuestas A= 0 de la B a la E= 1 } \\
\text { A. Nunca / raramente } \\
\text { B. De vez en cuando } \\
\text { C. A veces } \\
\text { D. Usualmente- casi siempre } \\
\text { E. Todo el tiempo - siempre }\end{array}$ \\
\hline
\end{tabular}


Tabla 2. Porcentaje de acuerdo de los jueces para cada ítem

\begin{tabular}{c|l|l}
\hline No ítem & \multicolumn{1}{|c}{ ítem español } & $\begin{array}{c}\text { \%de } \\
\text { acuerdo }\end{array}$ \\
\hline 1 & ¿A veces se olvida de tomar su medicamento? & 80 \\
\hline 2 & $\begin{array}{l}\text { La gente a veces omite tomar sus medicamentos por razones diferentes al olvido. Pen- } \\
\text { sando en las últimas } 2 \text { semanas, ¿hubo algún día que no toma su medicamento? }\end{array}$ & 100 \\
\hline 3 & $\begin{array}{l}\text { ¿Alguna vez ha recortado o dejado de tomar el medicamento sin consultar antes con su } \\
\text { médico, ya que se sintió peor cuando lo tomaba? }\end{array}$ & 80 \\
\hline 4 & Cuando viaja o sale de casa, ¿a veces se olvida de llevar su medicina? & 100 \\
\hline 5 & Ayer, ¿tomó todos los medicamentos? & 100 \\
\hline 7 & $\begin{array}{l}\text { Cuando siente que sus síntomas están bajo control, ¿a veces deja de tomar su medicamento? } \\
\text { Tomar medicamentos todos los días es un verdadero inconveniente para algunas perso- } \\
\text { nas. ¿Alguna vez se siente presionado a apegarse a su plan de tratamiento? }\end{array}$ & 100 \\
\hline 8 & $\begin{array}{l}\text { ¿Con qué frecuencia tiene dificultad para acordarse de tomar todos sus medicamentos? } \\
\text { Respuestas A= 0 de la B a la E= 1 } \\
\text { A. Nunca / raramente } \\
\text { B. De vez en cuando } \\
\text { C. A veces } \\
\text { D. Usualmente- casi siempre } \\
\text { E. Todo el tiempo - siempre }\end{array}$ & 80 \\
\hline
\end{tabular}

\section{Validez por juicio de expertos}

Con los datos obtenidos en la primera ronda del juicio de expertos se calculó el porcentaje de acuerdo entre los expertos para cada ítem encontrando que para algunos ítems este porcentaje estaba por debajo del $80 \%$ que se consideró bajo aunque la mayoría de las preguntas fueron catalogadas por los expertos como en alto y mediano grado de congruencia. En la segunda ronda algunos jueces manifestaron su decisión de cambiar algunas de las calificaciones a los diferentes ítems obteniéndose un porcentaje de acuerdo igual o mayor al $80 \%$ para todas las preguntas (tabla 2 ).

Con la información de la segunda ronda se calculó el coeficiente de concordancia w de Kendall que en este caso fue 0,8 con un $\mathrm{p}$ valor de 0,0001 y se interpretó como un alto grado de concordancia entre las valoraciones hechas por los jueces a los diferentes ítems del cuestionario. Considerando así que la escala de medición de adherencia al tratamiento de Morisky de 8 items es válida para medir la adherencia al tratamiento farmacológico de pacientes con enfermedad renal crónica según juicio de expertos.

\section{Prueba piloto}

Se aplicó el MMAS-8 a 30 pacientes con una media de edad de 75,9 años (+/- 15), un mínimo de edad de 54 años y un máximo de 90 años, en su mayoría hombres (62\%), dentro de las comorbilidades más frecuentes están la Hipertensión arterial $(90,6 \%)$ seguida de la diabetes mellitus (25\%); Los estadios de enfermedad renal crónica más frecuentes de los pacientes encuestados fueron $3 \mathrm{~b}(34,4 \%)$ y $4(34,4 \%)$, con estrato socioeconómico en su mayoría Nivel 3 el $53 \%$ y Nivel educativo de secundaria en más del $50 \%$ (tabla 3). 
Tabla 3. Características Sociodemográficas de la población $(N=30)$

\begin{tabular}{|c|c|}
\hline & $\%$ \\
\hline \multicolumn{2}{|l|}{ Género } \\
\hline Masculino & 62,5 \\
\hline Femenino & 37,5 \\
\hline \multicolumn{2}{|l|}{ Edad } \\
\hline$<59$ & 3,2 \\
\hline $60-74$ & 34,3 \\
\hline$>75$ & 62,5 \\
\hline \multicolumn{2}{|l|}{ Nivel educativo } \\
\hline Ninguno & 6,3 \\
\hline Primaria & 12,5 \\
\hline Secundaria & 59,4 \\
\hline Técnico & 6,3 \\
\hline Universitarios & 15,6 \\
\hline \multicolumn{2}{|l|}{ Zona de vivienda } \\
\hline Rural & 22,6 \\
\hline Urbana & 77,4 \\
\hline \multicolumn{2}{|c|}{ Evaluación socioeconómica } \\
\hline Nivel 1 & 3,1 \\
\hline Nivel 2 & 12,5 \\
\hline Nivel 3 & 53,1 \\
\hline Nivel 4 & 28,1 \\
\hline Nivel 5 & 3,1 \\
\hline \multicolumn{2}{|l|}{ Comorbilidades } \\
\hline Diabetes Mellitus & 25 \\
\hline Hipertensión arterial & 90,6 \\
\hline Glomerulopatias & 3,1 \\
\hline Cáncer & 15,6 \\
\hline
\end{tabular}

El 65,6\% era independiente según la escala de Barthel. Los niveles de albúmina se encontraban en rango normal en el $96 \%$ de los pacientes y los de hemoglobina en metas por encima del $70 \%$ tanto en hombres como en mujeres. El número de medicamentos por día fue de dos a cinco en el $43 \%$ de los pacientes y de seis a diez en el $40,6 \%$ de los casos. (Tabla 4).

El nivel de adherencia al tratamiento se analizó según el puntaje de la escala de Morisky de 8 items, clasificándo-
Tabla 4. Características clínicas de los pacientes $(N=30)$

\begin{tabular}{|l|c|}
\hline $\begin{array}{c}\text { Tasa de filtración glomerular } \\
\mathrm{ml} / \mathrm{min} / \mathrm{m} 2 \mathrm{sc}\end{array}$ & $\%$ \\
\hline Estadio $5(<15)$ & 0 \\
\hline Estadio $4(15-30)$ & 34,3 \\
\hline Estadio 3b (30 - 35) & 46,8 \\
\hline Estadio $3 \mathrm{a}(35-60)$ & 15,6 \\
\hline Estadio $2(>60)$ & 3,12 \\
\hline Hemoglobina Hombres & \\
\hline$>13 \mathrm{mg} / \mathrm{dL}$ & 75 \\
\hline $10-12,9 \mathrm{mg} / \mathrm{dL}$ & 25 \\
\hline$<10 \mathrm{mg} / \mathrm{dL}$ & 0 \\
\hline Hemoglobina mujeres & \\
\hline$>12 \mathrm{mg} / \mathrm{dL}$ & 83 \\
\hline $10-11,9 \mathrm{mg} / \mathrm{dL}$ & 8,3 \\
\hline$<10 \mathrm{mg} / \mathrm{dL}$ & 8,3 \\
\hline Albúmina & \\
\hline$>3,5 \mathrm{gr} / \mathrm{dL}$ & 96,8 \\
\hline
\end{tabular}

se como baja adherencia al tratamiento en el $62,5 \%$ de los casos con puntaje menor de 6 , mediana adherencia $28,1 \%$ de los pacientes puntaje entre 6 y 7 y alta adherencia solo el 9,4\% de los pacientes con puntaje mayor de 8 .

\section{Discusión}

Este es el primer estudio para validar la MMAS-8 en pacientes colombianos con enfermedad renal crónica, sometiendo la encuesta a juicio de expertos, teniendo en cuente que ya ha sido validada en diferentes idiomas y patologías crónicas, en Francia para hipertensión arterial, en corea y malasia para pacientes con diabetes tipo 2 y en pacientes que toman warfarina en Singapur (11,16-19). La traducción simple no es suficiente cuando se trata de cuestionarios que serán utilizados en los servicios de salud, ya que la validación y adaptación cultural es necesaria para preservar las propiedades psicométricas del instrumento que en este caso ya han sido validadas en diferentes idiomas. En aras de que esta nueva versión de MMAS-8 sea similar a la original en inglés el 
proceso de validación y adaptación guarda relación con lo recomendado por Ramada y colaboradores (20).

En cuanto a los pacientes encuestados en la prueba piloto, exhiben características sociodemográficas diferentes a lo descrito en la literatura para pacientes con enfermedad renal crónica en la población colombiana, en este estudio la mayoría de pacientes reciben de 1 a 5 medicamentos al día, predominó el sexo masculino y la edad promedio estuvo cercana a los 60 años y pertenecían a estrato socioeconómico 3 considerado como medio, Estas diferencias pueden explicarse porque este estudio se realizó en la unidad renal de una institución de salud que presta servicios exclusivamente a la población adscrita las fuerzas militares de Colombia (21).

Con respecto a la adherencia al tratamiento llama la atención que más del $70 \%$ de la población estudiada cumple con las metas de tratamiento si tenemos en cuenta las variables clínicas y de laboratorio que fueron tenidas en cuenta en este trabajo, sin embargo el $62,5 \%$ de los pacientes fueron clasificados como en baja adherencia al tratamiento y solo el 9,4\% de los pacientes clasificaron como alta adherencia al tratamiento, comparado con otros estudios en los cuales el porcentaje de baja adherencia fue del 24,8\% (22), que también reportaron que patologías como la EPOC y la Diabetes tenían menor adherencia que el VIH y la artritis, lo que nos da un amplio panorama en el cual podemos encaminar trabajos de investigación con el fin de identificar cuáles son las principales causas de baja adherencia en los pacientes con enfermedad renal crónica.

En conclusión la escala de Morisky de 8 items traducida al español es válida para medir la adherencia al tratamiento farmacológico en pacientes con enfermedad renal crónica.

En el presente estudio la encuesta de Morisky de 8 items traducida al español, mostro un buen potencial como herramienta de detección en la práctica clínica para identificar pacientes con enfermedad renal crónica en el programa de nefroprotección no adherentes al tratamiento.

\section{Agradecimientos}

A la universidad Militar Nueva Granada a la facultad de Medicina porque este es un producto derivado del proyecto MED 1797 financiado por la Vicerrectoría de Investigaciones de la UMNG Vigencia 2015. Al Hospital Militar Central por permitirnos trabajar con la unidad Re- nal, Al Dr. Donald Morisky por su amable colaboración en el proceso de traducción y adaptación de la MMAS-8.

\section{Conflictos de intereses}

Los autores declaran no tener conflicto de intereses, la financiación de esta investigación estuvo a cargo de la vicerrectoría de investigaciones de la UMNG, proyecto MED 1797.

\section{Referencias}

1. KDIGO 2012 Clinical Practice Guideline for the Evaluation and Management of Chronic Kidney Disease. Chapter 2: Definition, identification, and prediction of CKD progression. Kidney Int Supplements 2013; 3:63-72.

2. Avila Nelly .Enfermedad renal crónica: prevención y detección temprana en el primer nivel de atención. Med Int Mex.2013; 29:148-153.

3. Instituto Nacional de Salud, Observatorio Nacional de Salud, Primer Informe ONS, aspectos relacionados con la frecuencia de uso de los servicios de salud, mortalidad y discapacidad en Colombia, 2011. Imprenta Nacional de Colombia, Bogotá, D.C., Colombia 2013.

4. Ruilope LM, Salvetti A, Jamerson K, Hansson L, Warnold $\mathrm{I}$, Wedel $\mathrm{H}$, et al .Renal function and intensive lowering of blood pressure in hypertensive participants of the hypertension optimal treatment (HOT) study. J Am Soc Nephrol 2001; 12:218-225.

5. Bayés B, Romero R. Enfermedad cardiovascular e insuficiencia renal crónica. Med Clin. 2008; 131(2):60-2.

6. Martin de Francisco AL, Aguilera L, Fuster V. Enfermedad cardiovascular, enfermedad renal y otras enfermedades crónicas. Es necesaria una intervención más temprana en la enfermedad renal crónica. Aten Primaria. 2009; 41(9):511-514.

7. Sánchez M, Toro P, Perez M, Gomez AM, Portolés JM. Intervención farmacéutica en la adherencia al tratamiento de pacientes con enfermedad renal crónica. Rev Calid Asist. 2011; 26(3):146-151.

8. Tercer Consenso de Granada sobre Problemas Relacionados con Medicamentos (PRM) y resultados negativos asociados a la medicación (RNM). Ars Pharmaceutical. 2007; 48:5-17.

9. Sajatovic M, Velligan DI, Weiden PJ, Valenstein MA, Ogedegbe G. J Psychosom Res. 2010; 69: 591-599.

10. Morisky DE, Ang A, Krousel-Wood M, Ward HJ.Predictive validity of a medication adherence measure in an outpatient setting. J Clin Hypertens .2008; 5:348-354.

11. Korb-Savoldelli V, Gillaizeau F, Pouchot J, Lenain E, Postel-Vinay N, Plouin PF, Durieux P, Sabatier B. Validation of a French version of the 8-item Morisky medication adher- 
ence scale in hypertensive adults. J Clin Hypertens.2012; 14(7):429-34.

12. Wang J, Bian RW, Yong-zhen Mo. Validation of the Chinese version of the eight-item Morisky medication adherence scale in patients with type 2 diabetes mellitus. J Clin Gerontology \& Geriatrics 2013; 1(4):119-122.

13. Diaz A, Donald E. Morisky, Felizardo N, Costa ,Divaldo Pereira de Lyra. The 8-item Morisky Medication Adherence Scale: Validation of a Brazilian-Portuguese version in hypertensive adults. Research in Social and Administrative Pharmacy.2014; 10(3): 554 - 561.

14. Yang A,Wang B, Zhu G, Jiao Z,Fang J, et al. Validation of Chinese version of the Morisky Medication Adherence Scale in patients with epilepsy.Seizure.2014; 23(4):295299.

15. Harkness JA, Schoua-glusberg A. Questionnaires in Translation. ZUMA-Nachrichten Spezial, January 1998. Disponible en: http://isites.harvard.edu/fs/docs/icb.topic506406.files/znspez3_04_Harkness_Glusberg.pdf.

16. Lee WY, Ahn J, Kim JH, Hong YP, Hong SK, Kim YT, et al. Reliability and validity of a self-reported measure of medication adherence in patients with type 2 diabetes mellitus in Korea. J Int Med Res.2013; 41: 1098-110.

17. Al-Qazaz H, Hassali MA, Shafie AA, Sulaiman SA, Sundram S, Morisky DE. The eight-item Morisky Medication Adherence Scale MMAS: translation and vali-dation of the Malaysian version. Diabetes Res Clin Pract. 2010; 90:216-21.
18. Reynolds K, Viswanathan HN, O'Malley CD, Muntner P, Harrison TN, Cheetham TC, et al. Psychometric properties of the Osteoporosis-specific Morisky Medi-cation Adherence Scale in postmenopausal women with osteoporosis newly treated with bisphosphonates. Ann Pharmacother.2012; 46:659-70.

19. Wang Y, Kong MC, Ko Y. Psychometric properties of the 8-item Morisky Medication Adherence Scale in patients taking warfarin. Thromb Haemost. 2012; 108:789-95.

20. Ramada-Rodilla J.M, Serra-Pujadas C, Delclós-Clanchet G.L. Adaptación cultural y validación de cuestionarios de salud: revisión y recomendaciones metodológicas. Rev de Salud Public Mex . 2013; 55(1).

21. Disponible en: http://www.scielo.org.mx/scielo.php?pid =S0036-342013000100009\&script=sci_arttext

22. Contreras F, Esguerra G.A., Espinosa J.C, Gómez V. Estilos de afrontamiento y calidad de vida en pacientes con insuficiencia renal crónica (IRC) en tratamiento de hemodiálisis.Act.Colom.Psicol.2007; 10(2):169-179 Disponible en http://www.scielo.org.co/pdf/acp/v10n2/ v10n2a16.pdf 